\title{
Pseudo Boolean Programming for Partially Ordered Genomes
}

\author{
Sébastien Angibaud ${ }^{1}$, Guillaume Fertin ${ }^{1}$, Annelyse Thévenin $^{2}$ and Stéphane \\ Vialette $^{3}$ \\ ${ }^{1}$ Laboratoire d'Informatique de Nantes-Atlantique (LINA), UMR CNRS 6241 \\ Université de Nantes, 2 rue de la Houssinière, 44322 Nantes Cedex 3 - France \\ ${ }^{2}$ Laboratoire de Recherche en Informatique (LRI), UMR CNRS 8623 \\ Université Paris-Sud, 91405 Orsay - France \\ 3 IGM-LabInfo, UMR CNRS 8049, Université Paris-Est, \\ 5 Bd Descartes 77454 Marne-la-Vallée, France \\ \{Sebastien.Angibaud, Guillaume.Fertin\}@univ-nantes.fr, thevenin@lri.fr, \\ vialette@univ-mlv.fr
}

\begin{abstract}
Comparing genomes of different species is a crucial problem in comparative genomics. Different measures have been proposed to compare two genomes: number of common intervals, number of adjacencies, number of reversals, etc. These measures are classically used between two totally ordered genomes. However, genetic mapping techniques often give rise to different maps with some unordered genes. Starting from a partial order between genes of a genome, one method to find a total order consists in optimizing a given measure between a linear extension of this partial order and a given total order of a close and well-known genome. However, for most common measures, the problem turns out to be NP-hard. In this paper, we propose a $(0,1)$-linear programming approach to compute a linear extension of one genome that maximizes the number of common intervals (resp. the number of adjacencies) between this linear extension and a given total order. Next, we propose an algorithm to find linear extensions of two partial orders that maximize the number of adjacencies.
\end{abstract}

\section{Introduction}

Genetic mapping techniques often give rise to different maps with some unordered genes. In that case, maps are combined in the form of a partially ordered sequence of genes, and thus a genome is modeled by a poset (i.e., a partially ordered set), as was done in [10]. In this model, any linear extension of a poset represents a possible total order of the genome. In order to find a total order from a partial order, Sankoff et al. [10] suggested the following method: confront the partial order with a given total order of a close and well-known genome. More precisely, this method asks for a linear extension of the partially ordered genome that optimizes a (dis)similarity measure between this total order and a given totally ordered genome. Several measures can be used: number of common intervals [8], of adjacencies [1], of breakpoints [9], of conserved intervals [3], etc. 
Computing these measures between two total orders, whenever no gene is duplicated, is a polynomial-time solvable problem. Unfortunately, concerning the number of adjacencies, the number of common intervals and the reversal distance, finding a linear extension that optimizes one of these measures between this extension and a given total order is NP-hard [4,6]. In this paper, we first present an approach to compute a linear extension of a partially ordered genome with respect to a given totally ordered genome without duplicated genes. Our method lies on a transformation of the initial problem into a $(0,1)$-linear program (i.e., a linear program with boolean variables) [2,1]. We focus here on two similarity measures: the number of common intervals and the number of adjacencies. We also extend our approach and consider the problem of comparing two partially ordered genomes with respect to the number of adjacencies. After presenting our three algorithms, we evaluate our method by using the same simulated data as in [4].

This paper is organized as follows. In Section 2, we present some preliminaries and definitions. We focus in Section 3 on the problem of finding a total order of a partially ordered genome that maximizes either the number of common intervals or the number of adjacencies with a given totally ordered genome. Next, we extend the case of adjacencies to find two totally ordered genomes from two partially ordered genomes. For each problem, we give its formulation in terms of a $(0,1)$-linear program, together with some reduction rules that ail at speeding-up the process. Section 4 is devoted to experimental results on simulated data.

\section{Preliminaries}

In the literature, a (totally ordered) duplication-free genome having $n$ oriented genes is usually represented by a signed permutation, one where each element in the set $\{1,2, \ldots, n-1, n\}$ has either a sign, + or - . Let $T_{1}$ and $T_{2}$ be two duplication-free genomes of size $n$. Wlog, we may assume that $T_{2}$ is the identity positive permutation, i.e., $T_{2}=+1+2 \ldots+n$. An interval of $T_{1}$ is a set of $m$ consecutive genes $\left\{\mathrm{g}_{1}, \mathrm{~g}_{2}, \ldots, \mathrm{g}_{m}\right\}$ in $T_{1}$. A common interval between $T_{1}$ and $T_{2}$ is an interval which occurs in $T_{1}$ and in $T_{2}$. Observe that the notion of common interval does not take into account the sign of the genes. We say that there is a breakpoint after gene $T_{1}[i], 1 \leq i \leq n-1$, in $T_{1}$ if neither $T_{1}[i]$ and $T_{1}[i+1]$ nor $-T_{1}[i+1]$ and $-T_{1}[i]$ are consecutive genes in $T_{2}$, otherwise we say that there is an adjacency after gene $T_{1}[i]$. Notice, that in order to take into account common intervals and adjacencies that may occur at the extremities of a genome, we artificially add to a genome with $n$ genes the gene +0 (resp. $+(n+1))$ to its left (resp. right). We now introduce the required material for partially ordered genomes. A partial order on a set $P$ is a binary relation $\preceq$ such that, for $x, y$ and $z$ in $P$, (i) $x \preceq x$, (ii) $x \preceq y$ and $y \preceq x$ imply $x=y$, and (iii) $x \preceq y$ and $y \preceq z$ imply $x \preceq z$. These three relations are referred to as reflexivity, antisymmetry, and transitivity, respectively. A set $P$ equipped with a partial order relation is said to be a partially ordered set (also called a poset). 
When it is necessary to specify the partial order relation, we write $(P, \preceq)$. A partial order relation $\preceq$ on $P$ gives rise to a relation $\prec$ of strict inequality: $x \prec y$ in $P$ iff $x \preceq y$ and $x \neq y$. Strict partial orders differ from partial orders only in whether each element is required to be unrelated, or required to be related, to itself. A totally ordered set (or linearly ordered set) is a poset $P$ which has the property that every two elements of $\mathrm{P}$ are comparable (i.e., for all $x, y \in P$ either $x \preceq y$ or $y \preceq x$ ). A poset $Q$ is called an extension of a poset $P$ if the elements of $P$ and $Q$ are the same, and the set of relations of $P$ is a subset of the set of relations of $Q$ (i.e., for all $x, y \in P$, if $x \prec y$ in $P$, then $x \prec y$ in $Q$, but not necessarily conversely). $Q$ is called a linear extension of $P$ if $Q$ is an extension of $P$ and also a linear order. In our context, a totally (resp. partially) ordered genome is thus a set $P$ that contains exactly one occurrence of $i$ or $-i$, $0 \leq i \leq n+1$, equipped with some total (resp. partial) order. For the sake of brevity, the totally ordered genome $+0+1+2 \ldots+n+(n+1)$ is abbreviated as Id. Let $P_{1}$ and $P_{2}$ be two partially ordered genomes, and let $x \in\{1,2\}$. The sign of a gene $\mathrm{g}$ in $P_{x}$ is written $s_{x}(\mathrm{~g})$. We write $\mathrm{g}_{1} \prec_{x} \mathrm{~g}_{2}$ if gene $\mathrm{g}_{1}$ precedes gene $\mathrm{g}_{2}$ in $P_{x}$. The number of genes $\mathrm{g}_{1}$ such that $\mathrm{g}_{1} \prec_{x} \mathrm{~g}_{2}$ is denoted by $\operatorname{prec}_{x}\left(\mathrm{~g}_{2}\right)$. We write $\mathrm{g}_{2} \succ_{x} \mathrm{~g}_{1}$ if gene $\mathrm{g}_{2}$ follows $\mathrm{g}_{1}$ in $P_{x}$. The number of genes $\mathrm{g}_{2}$ such that $\mathrm{g}_{1} \prec_{x} \mathrm{~g}_{2}$ is denoted by $\operatorname{succ}_{x}\left(\mathrm{~g}_{1}\right)$. In $P_{x}$, two genes $\mathrm{g}_{1}$ and $\mathrm{g}_{2}$ are said to be incomparable (written $\mathrm{g}_{1} \|_{x} \mathrm{~g}_{2}$ ) if neither $\mathrm{g}_{1} \prec_{x} \mathrm{~g}_{2}$ nor $\mathrm{g}_{2} \prec_{x} \mathrm{~g}_{1}$. The width of a partially ordered genome is the size of a largest subset of incomparable genes. Two genes $\mathrm{g}_{1}$ and $\mathrm{g}_{2}$ are said to be adjoining in $P_{x}$ if there does not exist $\mathrm{g}_{3}$ such that $\mathrm{g}_{1} \prec_{x} \mathrm{~g}_{3} \prec_{x} \mathrm{~g}_{2}$ or $\mathrm{g}_{2} \prec_{x} \mathrm{~g}_{3} \prec_{x} \mathrm{~g}_{1}$ or $\mathrm{g}_{1} \|_{x} \mathrm{~g}_{3}$ or $\mathrm{g}_{2} \|_{x} \mathrm{~g}_{3}$. Let $T_{x}$ be a linear extension of $P_{x}$. The set of allowed positions of a gene $\mathrm{g}$ in $T_{x}$ is written by $P O S_{x}(\mathrm{~g})$; one can easily check that $P O S_{x}(\mathrm{~g})=\left\{\operatorname{prec}_{x}(\mathrm{~g})+1, \ldots, \operatorname{succ}_{x}(\mathrm{~g})-1\right\}$. The position of $\mathrm{g}$ in $T_{x}$ is written $T_{x}(\mathrm{~g})$ (by definition, $\left.T_{x}(\mathrm{~g}) \in P O S_{x}(\mathrm{~g})\right)$. We say that a gene $\mathrm{g}_{1}$ is $i$-nail $l_{x}$ if $P O S_{x}\left(\mathrm{~g}_{1}\right)=\{i\}$ (i.e., there are $i-1$ genes $\mathrm{g}_{2}$ such that $\mathrm{g}_{2} \prec_{x} \mathrm{~g}_{1}$ and no gene is incomparable with $\mathrm{g}_{1}$ in $P_{x}$ ). In this paper, we are interested in three combinatorial problems. The first two problems are concerned with confronting a partially ordered genome with a reference totally ordered genome: given a partially ordered genome $P_{1}$ and a reference totally ordered genome $T_{2}$, problem MCIL-1PO (resp. MAL-1PO) asks for a linear extension $T_{1}$ of $P_{1}$ that yields a maximum number of common intervals (resp. maximum number of adjacencies) between any linear extension of $P_{1}$ and $T_{2}$. Observe that there is no loss of generality in assuming here that $T_{2}=\mathrm{Id}$. The third problem is concerned with confronting two partially ordered genomes: given two partially ordered genomes $P_{1}$ and $P_{2}$, problem MAL-2PO asks for a linear extension $T_{1}$ of $P_{1}$ and a linear extension $T_{2}$ of $P_{2}$ s.t. the number of adjacencies between $T_{1}$ and $T_{2}$ is maximized among all linear extensions of $P_{1}$ and $P_{2}$. Note that problems MCIL-1PO, MAL-1PO and MAL-2PO have been proved to be NP-hard in [4].

\section{An exact $(0,1)$-linear programming approach}

We present in this section an exact generic approach. The main idea is to transform our problems into $(0,1)$-linear programs [7] and use a powerful solver to ob- 
tain optimal solutions. All computations conducted here use the minisat+ solver [5]. In order to solve problems MCIL-1PO, MAL-1PO and MAL-2PO, we present the $(0,1)$-linear programs called $\mathrm{CI}-1 \mathrm{PO}, \mathrm{Adj}-1 \mathrm{PO}$ and $\mathrm{Adj}-2 \mathrm{PO}$, respectively. Programs CI-1PO and Adj-1PO take as input a partial order $P_{1}$ while Adj-2PO takes two partial orders $P_{1}$ and $P_{2}$. We first present the common part of these three programs and next give a complete description of each program. Then, we present some data reduction rules for reducing the size of the programs.

The common part of the three programs. We present here the common part of programs CI-1PO, Adj-1PO and Adj-2PO. Let $P_{1}$ and $P_{2}$ be two partially ordered genomes over $[0: n+1]$. Fix $x$ to be 1 if we consider either CI-1PO or Adj-1PO, or 1 or 2 if we consider Adj-2PO. For each problem, we seek for a linear extension $T_{x}$ of $P_{x}$ that maximizes the number of common intervals or the number of adjacencies. Our programs are divided into two parts: (i) definition of a linear extension $T_{x}$, and (ii) maximization of the measure. The first part is common to the three programs whereas the second part is problem dependent and needs specific variables and constraints. To define a linear extension of a partially ordered genome, we use the same set $\mathcal{A}^{x}=\left\{a_{\mathrm{g}, i}^{x}: \mathrm{g} \in[0: n+1]\right.$ and $i \in[0$ : $n+1]\}$ of boolean variables. For each $a_{\mathrm{g}, i}^{x} \in \mathcal{A}^{x}, a_{\mathrm{g}, i}^{x}=1$ iff $T_{x}(\mathrm{~g})=i$, i.e., $\mathrm{g}$ is at position $i$ in the resulting linear extension. To this aim, we define the three constraints presented in Figure 1:

- (C.a) ensures that each gene is assigned to exactly one position in $T_{x}$,

- (C.b) ensures that no two genes are assigned to the same position in $T_{x}$,

- (C.c) checks genes order in $T_{x}$. Let $g_{1}$ and $g_{2}$ be two genes of $P_{x}$ such that $\mathrm{g}_{1} \prec_{x} \mathrm{~g}_{2}$. We must certainly check that $\mathrm{g}_{1}$ precedes $\mathrm{g}_{2}$ in $T_{x}$. Thus, for all $0 \leq j<i \leq n+1$ we impose that $a_{\mathrm{g}_{1}, i}^{x}=0$ or $a_{\mathrm{g}_{2}, j}^{x}=0$.

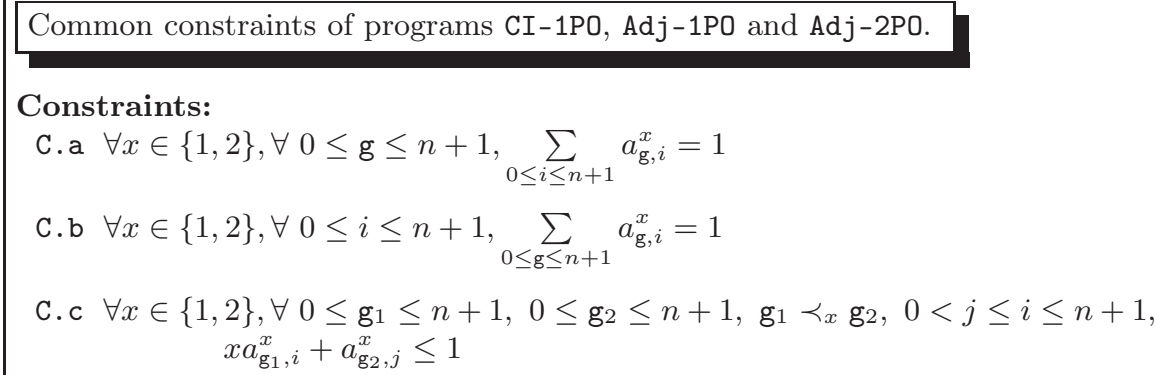

Fig. 1. Common constraints for CI-1PO, Adj-1PO and Adj-2PO. A gene $\mathrm{g}$ is at position $i$ in $T_{x}$ iff $a_{\mathrm{g}, i}^{x}$. Constraints C. a to C.c ensure that $T_{x}$ is a valid linear extension of $P_{x}$, $x \in\{1,2\}$.

Now, we present specific variables and constraints for each problem. 
Confronting a partially ordered genome with a totally ordered genome: maximizing the number of common intervals. We give here a $(0,1)$-linear program called CI-1P0 that computes a linear extension $T_{1}$ of a partially ordered genome $P_{1}$ that maximizes the number of common intervals between $T_{1}$ and Id. Recall that common intervals do not take into account the sign of the genes. Program CI-1PO is given in Figure 2.

We describe now the variables used in CI-1PO. Let $\mathrm{g} \in[0: n+1]$ be a gene, $i \in[0: n+1]$ be a position and $t \in[0: n+1]$. After having defined the total order $T_{1}$ with the set $\mathcal{A}^{1}$, we define two sets of boolean variables: $\mathcal{B}=\left\{b_{\mathrm{g}, i, t}: \mathrm{g} \in[0: n+1], i \in[0: n+1]\right.$ and $\left.t \in[0: n+1]\right\}$ and $\mathcal{C}=\left\{c_{\mathrm{g}, i, t}: \mathrm{g} \in[0: n+1], i \in[0: n+1]\right.$ and $\left.t \in[0: n+1]\right\}$. For each $b_{\mathrm{g}, i, t} \in \mathcal{B}$, we have $b_{\mathrm{g}, i, t}=1$ iff $T_{1}(\mathrm{~g}) \in[i: i+t]$, i.e., $\mathrm{g}$ is located between positions $i$ and $i+t$ in $T_{1}$. The set $\mathcal{C}$ corresponds to the set of all possible common intervals. For each $c_{\mathrm{g}, i, t} \in \mathcal{C}$, we have $c_{\mathrm{g}, i, t}=1$ iff, for all $k \in[0: t]$, we have $T_{1}(\mathrm{~g}+k) \in[i: i+t]$, i.e., the set of genes $\mathrm{g}+k(k \in[0: t])$ is an interval of $T_{1}$. In this case, we certainly have a common interval between $T_{1}$ and the identity. Therefore, the objective function is the sum of all variables $c_{\mathrm{g}, i, t} \in \mathcal{C}$.

Constraints (C.01) to (C.05) are needed to ensure correctness of CI-1PO. First, we obviously need constraints (C.01) to (C.03), that correspond to the common constraints (C.a) to (C.c), with $x=1$. Then, we add constraint (C.04) to check variables $b_{\mathrm{g}, i, t}: b_{\mathrm{g}, i, t}=1$ iff there exists $j$ in $[0: t]$ such that $a_{\mathrm{g}, i+j}=1$. Finally, we add constraint (C.05) to check variables $c_{\mathrm{g}, i, t}: c_{\mathrm{g}, i, t}=1 \mathrm{iff}$, for all $k$ in $[0: t]$, we have $b_{\mathrm{g}+k, i, t}=1$.

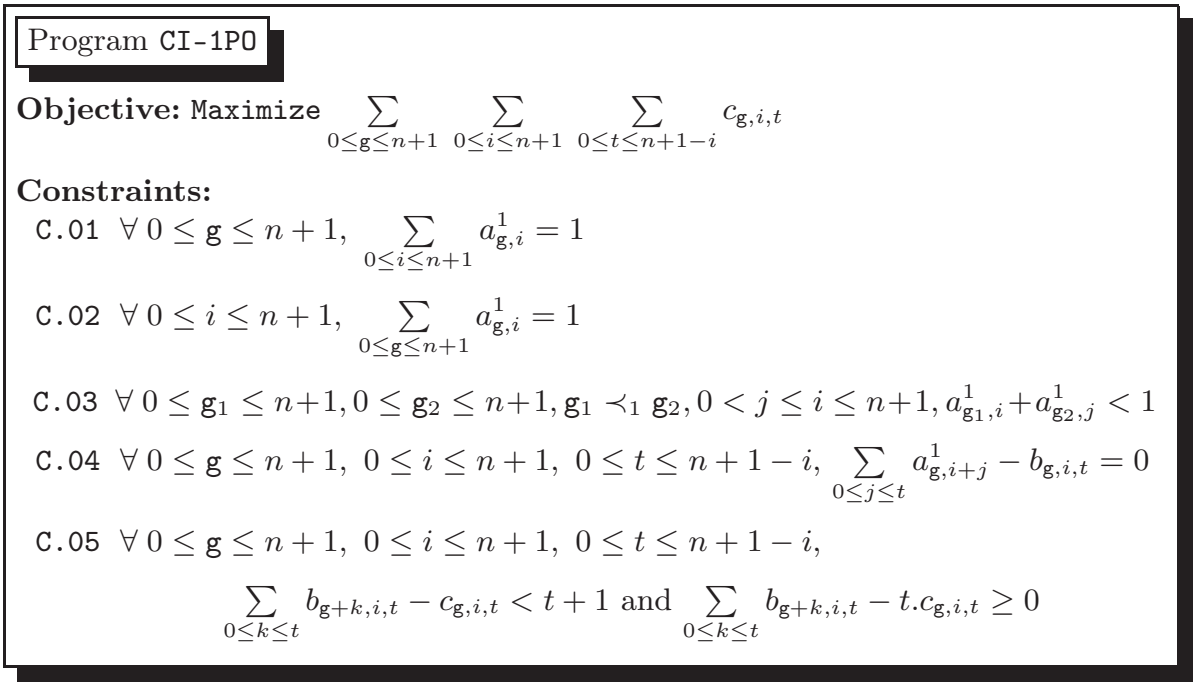

Fig. 2. Program CI-1PO computes a linear extension $L$ of a partial order maximizing the maximum number of common intervals between $L$ and the identity. 
Confronting a partially ordered genome with a totally ordered genome: maximizing the number of adjacencies. We now give a $(0,1)$-linear program (Adj-1PO) that computes a linear extension $T_{1}$ of a partial order $P_{1}$ that maximizes the number of adjacencies between $T_{1}$ and Id. Oppositely to common intervals, the sign of genes is now relevant. Program Adj-1PO is presented in Figure 3.

Let $\mathrm{g} \in[0: n+1]$ be a gene, and $i \in[0: n+1]$ be a position. After having defined the total order $T_{1}$ with the set of variables $\mathcal{A}^{1}$, we define the set $\mathcal{D}=\left\{d_{\mathrm{g}, i}: \mathrm{g} \in[0: n+1[\right.$ and $i \in[0: n+1]\}$ of boolean variables that corresponds to the set of possible adjacencies. For each $d_{\mathrm{g}, i} \in \mathcal{D}(\mathrm{g} \neq n+1)$, we have $d_{\mathrm{g}, i}=1$ iff (i) $T_{1}(\mathrm{~g})=i$ and (ii) $\mathrm{g}$ and $(\mathrm{g}+1)$ create an adjacency. Therefore, we define the objective function as the sum of all variables $d_{\mathrm{g}, i} \in \mathcal{D}$.

We now define the constraints $\left(\mathbf{C}^{\prime} . \mathbf{0 1}\right)$ to $\left(\mathbf{C}^{\prime} . \mathbf{0 6}\right)$ to ensure the validity of program Adj-1PO. We first add constraints ( $\left.C^{\prime} .01\right)$ to (C'.03), that correspond to the common constraints (C.a) to (C.c) with $x=1$, to obtain a valid linear extension $T_{1}$. Next, we add constraints (C'.04) to connect the assignment of variables in $\mathcal{D}$ to the assignment of variables in $\mathcal{A}^{1}$. To this aim, for each $d_{\mathrm{g}, i} \in \mathcal{D}$, $\mathrm{g} \neq n+1, i \notin\{0, n+1\}$, we have $d_{\mathrm{g}, i}=1$ iff the two following conditions hold: (i) $a_{\mathrm{g}, i}^{1}=1$ (i.e., $\mathrm{g}$ is at the position $i$ in $T_{1}$ ) and (ii) $a_{\mathrm{g}+1, i+1}^{1}=1$ (case 1 ) or $a_{\mathrm{g}+1, i-1}^{1}=1$ (case 2 ) (depending on the sign of $\mathrm{g}$ and $\mathrm{g}+1$ ). Finally, constraints $\left(\mathbf{C}^{\prime} \cdot 05\right)$ and $\left(\mathbf{C}^{\prime} .06\right)$ check variables $d_{\mathrm{g}, 0}$ and $d_{\mathrm{g}, n+1}$ that correspond to the two possible adjacencies located at the extremities. These two constraints are defined as constraints (C'.04), (C'.05) (case 1 only) and (C'.06) (case 2 only).

Confronting two partially ordered genomes: maximizing the number adjacencies. The $(0,1)$-linear program Adj-2PO proposed here computes two linear extensions $T_{1}$ and $T_{2}$ of two partially ordered genomes $P_{1}$ and $P_{2}$ such that the number of adjacencies between $T_{1}$ and $T_{2}$ is maximized. Recall that the sign of the genes is relevant here. Oppositely to MAL-1PO where one genome is totally ordered, we do not know the relation between two genes that will eventually create an adjacency. Program Adj-2PO is given in Figure 4.

Let $\mathrm{g}_{1} \in[0: n+1]$ and $\mathrm{g}_{2} \in[0: n+1]$ be two genes, and let $i \in[0: n+1]$ and $j \in[0: n+1]$ be two positions. After having defined the total orders $T_{1}$ and $T_{2}$ with the sets of variables $\mathcal{A}^{1}$ and $\mathcal{A}^{2}$, we introduce the set of variables $\mathcal{E}=\left\{e_{\mathrm{g}_{1}, i, j, \mathrm{~g}_{2}}: \mathrm{g}_{1} \in[0: n+1], i \in[0: n+1], j \in[0: n+1]\right.$ and $\left.\mathrm{g}_{2} \in[0: n+1]\right\}$ that correspond to the possible adjacencies. A boolean variable $e_{\mathrm{g}_{1}, i, j, \mathrm{~g}_{2}} \in \mathcal{E}$ has of four indices: genes $\mathrm{g}_{1}$ and $\mathrm{g}_{2}$, and positions $i$ and $j$. For each $e_{\mathrm{g}_{1}, i, j, \mathrm{~g}_{2}} \in \mathcal{E}$, we have $e_{\mathrm{g}_{1}, i, j, \mathrm{~g}_{2}}=1$ iff (i) genes $\mathrm{g}_{1}$ and $\mathrm{g}_{2}$ create an adjacency and (ii) $T_{1}\left(\mathrm{~g}_{1}\right)=i$, $T_{2}\left(\mathrm{~g}_{1}\right)=j$, and $T_{1}\left(\mathrm{~g}_{2}\right)=i+1$. The objective function is defined as the sum of variables $e_{\mathrm{g}_{1}, i, j, \mathrm{~g}_{2}} \in \mathcal{E}$.

We define constraints (C'.01) to (C'.09) to check correctness of program Adj-2PO. First, we use again the common constraints (C.a) to (C.c). Here, we must use these constraints for $x$ in $\{1,2\}$ in order to define two linear extensions. We refer to these constraints as constraints $(C " .01)$ to $(C " .06)$. Then, we check the assignment of variables $e_{\mathrm{g}_{1}, i, j, \mathrm{~g}_{2}}$ for $i \neq n+1$ and $j \notin\{0, n+1\}$ with constraint (C'.07). To this aim, for each $e_{\mathrm{g}_{1}, i, j, \mathrm{~g}_{2}}$, we have $e_{\mathrm{g}_{1}, i, j, \mathrm{~g}_{2}}=1$ iff the two following conditions hold: (i) $a_{\mathrm{g}_{1}, i}^{1}=1, a_{\mathrm{g}_{1}, j}^{2}=1$, and $a_{\mathrm{g}_{2}, i+1}^{1}=1$, and (ii) $a_{\mathrm{g}_{2}, j+1}^{2}=1$ 


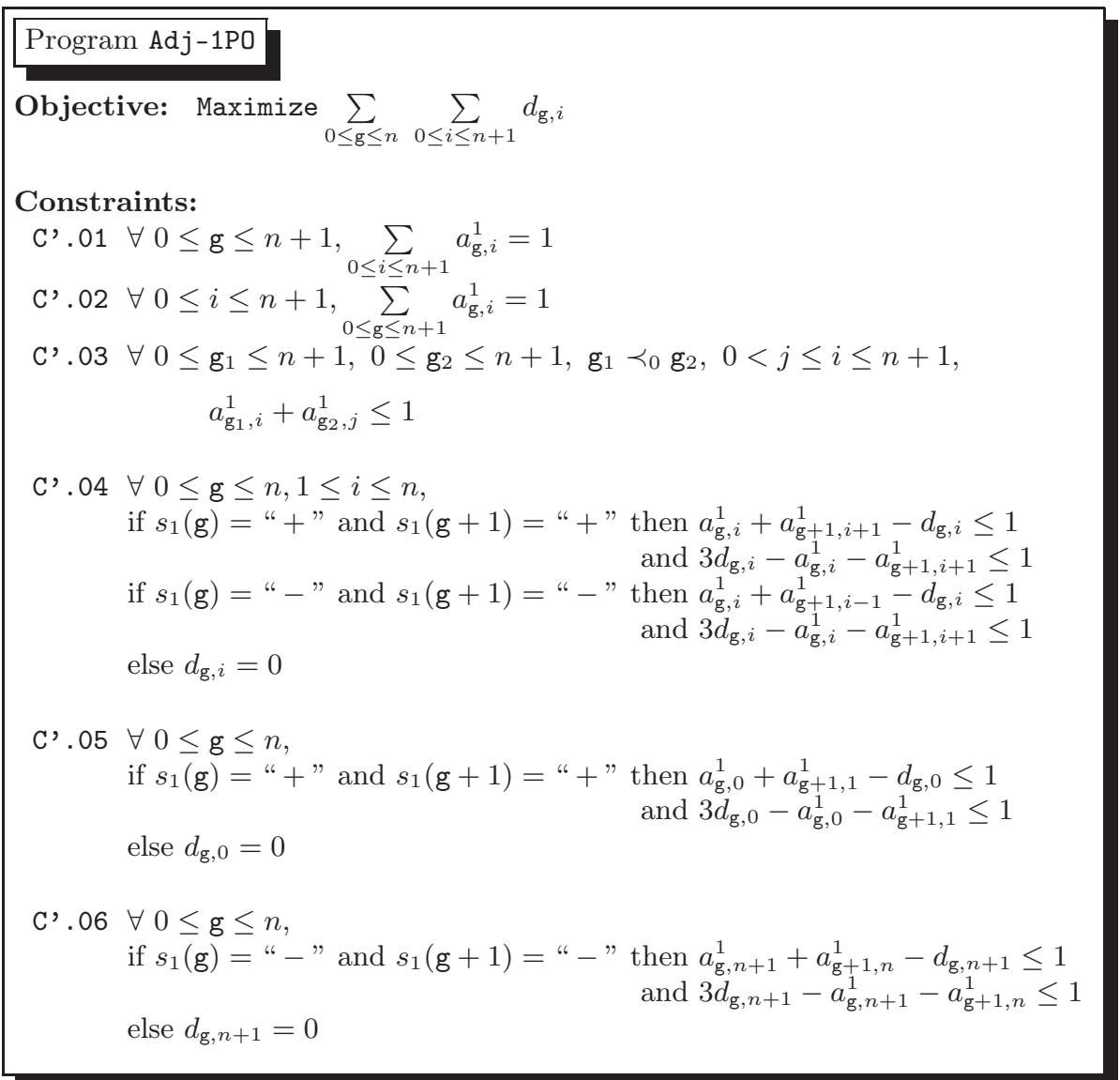

Fig. 3. Program Adj-1PO computes a linear extension of a partially ordered genome that maximizes the number of adjacencies between this linear extension and the identity.

(case 1 ) or $a_{\mathrm{g}_{2}, j-1}^{2}=1$ (case 2) (these two cases depend on the sign of $\mathrm{g}_{1}$ and $\left.\mathrm{g}_{2}\right)$. Finally, we add constraints $\left(\mathbf{C}^{\prime} \cdot \mathbf{0 8}\right)$ and $\left(\mathrm{C}^{\prime} \cdot \mathbf{0 9}\right)$ to ensure the validity of variables $e_{\mathrm{g}_{1}, i, 0, \mathrm{~g}_{2}}$ and $e_{\mathrm{g}_{1}, i, n+1, \mathrm{~g}_{2}}$ that correspond to possible adjacencies located at the extremities. These two constraints are similar to (C'.07) but constraints (C'.08) is for case 1 only and (C'.09) is for case 2 only.

Speeding-up the program. Program CI-1PO has $O\left(n^{3}\right)$ variables and $O\left(n^{3}\right)$ constraints. Program Adj-1PO has $O\left(n^{2}\right)$ variables and $O\left(n^{2}\right)$ constraints, while program Adj-2PO has $O\left(n^{4}\right)$ variables and $O\left(n^{4}\right)$ constraints. In order to speedup the running time of the programs, we present here some simple rules for reducing the number of variables and constraints involved in the programs or for computing in a fast preprocessing step some of the variables. 


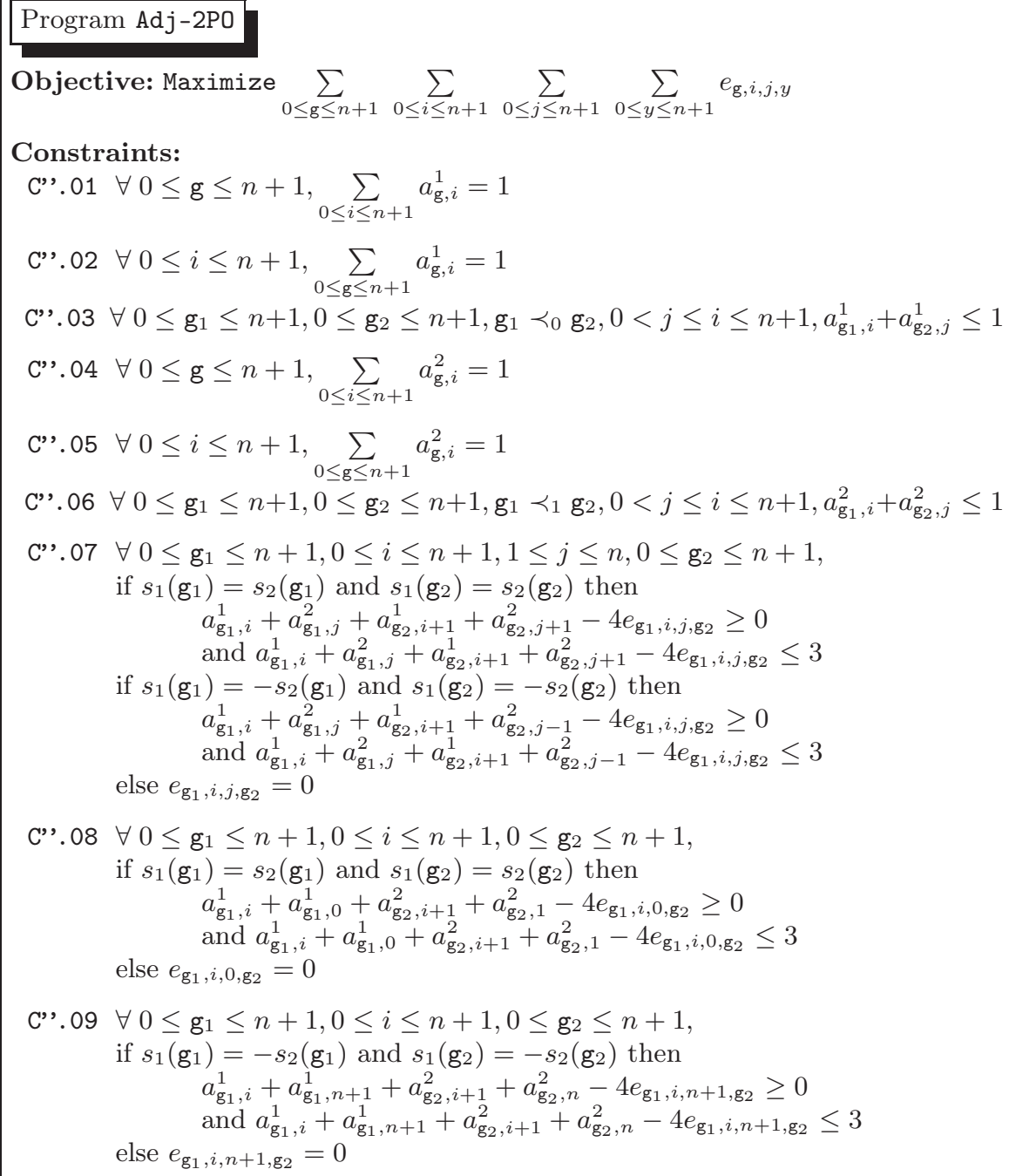

Fig. 4. Program Adj-2PO computes two linear extensions of two partial orders maximizing the number of adjacencies.

Restricted position. In $T_{x}$, for each gene $\mathrm{g}, T_{x}(\mathrm{~g})$ must be in $P O S_{x}(\mathrm{~g})$. According to this remark, some variables can be pre-computed:

- $\forall x \in\{1,2\}, \forall a_{\mathrm{g}, i}^{x} \in \mathcal{A}^{x}, a_{\mathrm{g}, i}^{x}=0$ if $i \notin P O S_{x}(\mathrm{~g}) ;$

- $\forall b_{\mathrm{g}, i, t} \in \mathcal{B}, b_{\mathrm{g}, i, t}=0$ if $T_{1}(\mathrm{~g})$ cannot be in $[i: i+t]$, and $b_{\mathrm{g}, i, t}=1$ if $T_{1}(\mathrm{~g})$ is necessarily in $[i: i+t]$; 
$-\forall d_{\mathrm{g}, i} \in \mathcal{D}, d_{\mathrm{g}, i}=0$ if $i \notin P O S_{1}(\mathrm{~g})$

- $\forall e_{\mathrm{g}_{1}, i, j, \mathrm{~g}_{2}} \in \mathcal{E}, e_{\mathrm{g}_{1}, i, j, \mathrm{~g}_{2}}=0$ if one of the four following conditions holds: (i) $i \notin P O S_{1}\left(\mathrm{~g}_{1}\right)$, (ii) $i+1 \notin P O S_{1}\left(\mathrm{~g}_{2}\right)$, (ii) $i+1 \notin P O S_{2}\left(\mathrm{~g}_{2}\right)$ and $s_{1}\left(\mathrm{~g}_{1}\right)=$ $s_{1}\left(\mathrm{~g}_{2}\right)$, (iv) $i-1 \notin P O S_{2}\left(\mathrm{~g}_{2}\right)$ and $s_{1}\left(\mathrm{~g}_{1}\right)=-s_{1}\left(\mathrm{~g}_{2}\right)$.

For each gene $\mathrm{g}$ that is $i$-nail $l_{x}$ (i.e., $\mathrm{g}$ is necessarily at a known position $i$ in the linear extension), some variables can also be pre-computed:

- $\forall x \in\{1,2\}, \forall a_{\mathrm{g}, i}^{x} \in \mathcal{A}^{x}, a_{\mathrm{g}, i}^{x}=1$ if $\mathrm{g}$ is $i$-nail ${ }_{x}$

$-\forall d_{\mathrm{g}, i} \in \mathcal{D}, d_{\mathrm{g}, i}=1$ if gene $\mathrm{g}$ is $i$-nail 1 and if one of the following conditions is satisfied: (i) gene $(\mathrm{g}+1)$ is $(i+1)-n a i l_{2}$ with $s_{1}(\mathrm{~g})=$ "+" and $s_{1}(\mathrm{~g}+1)=$ "+", or (ii) gene $(\mathrm{g}+1)$ is $(i-1)-$ nail 2 with $s_{1}(\mathrm{~g})=$ " - " and $s_{1}(\mathrm{~g}+1)="-"$.

- $\forall e_{\mathrm{g}_{1}, i, j, \mathrm{~g}_{2}} \in \mathcal{E}, e_{\mathrm{g}_{1}, i, j, \mathrm{~g}_{2}}=1$ if gene $\mathrm{g}_{1}$ is $i$-nail $l_{1}$ and $j$-nail ${ }_{2}$, gene $\mathrm{g}_{2}$ is a $(i+1)-$ nail $_{1}$, and one of the two following conditions holds: (i) $\mathrm{g}_{2}$ is $(j+1)$ nail 2 with $s_{1}\left(\mathrm{~g}_{1}\right)=s_{1}\left(\mathrm{~g}_{2}\right)$, (ii) $\mathrm{g}_{2}$ is $(j-1)$-nail 2 with $s_{1}\left(\mathrm{~g}_{1}\right)=-s_{1}\left(\mathrm{~g}_{2}\right)$.

Specific rules for CI-1PO. By definition, each interval of size 1 and $n+2$ is a common interval. Therefore, $c_{\mathrm{g}, i, 0}=1$ and $c_{\mathrm{g}, 0, n+2}=1$ for $0 \leq \mathrm{g} \leq n+1$, and we add $n+2$ to the objective function. Also, we can delete all variables $c_{\mathrm{g}, i, 0}$ and $c_{\mathrm{g}, i, n+1}$ in the program as well as their related constraints. The second reduction consists in removing some variables $c_{\mathrm{g}, i, t}$. We do not generate a variable $c_{\mathrm{g}, i, t}$ and its related constraints if $c_{\mathrm{g}, i, t}=1$ is not possible. Indeed, if there exists an integer $k \in[0: t]$ such that $T_{1}(\mathrm{~g}+k)$ cannot be in $[i: i+t]$ (i.e., gene $\mathrm{g}+k$ cannot be between $i$ and $i+t$ in the linear extension), then the interval $\{\mathrm{g}, \mathrm{g}+1, \ldots, \mathrm{g}+t\}$ is certainly not a common interval.

Specific rules for Adj-1PO. Constraint $C^{\prime} .06$ defines the adjacency created by genes $\mathrm{g}$ and $(\mathrm{g}+1)$ at positions $n+1$ and $n$, respectively, in $T_{1}$. Since we have artificially added gene $n+1$ at the end of partial order, then the gene at position $n+1$ is $n+1$. Therefore, $d_{\mathrm{g}, n+1}=0$ for $\mathrm{g}$ in $[0: n]$ and the constraints $\mathrm{C}^{\prime} .06$ are deleted. Moreover, if no adjacency is possible between two genes that are nails, then the total order between these genes has no impact on the number of adjacencies. Specifically, let $\mathrm{g}_{1}$ and $\mathrm{g}_{2}$ be two genes such that $\mathrm{g}_{1}$ is $i_{1}$-nail $l_{1}$ and $\mathrm{g}_{2}$ is $i_{2}$-nail $l_{1}\left(i_{1}<i_{2}\right)$. Notice that no adjacency can be create between the positions $i_{1}$ and $i_{2}$ between a linear extension of $P_{1}$ and the identity if, for each gene $\mathrm{g}_{3}$ such that $P O S_{1}\left(\mathrm{~g}_{3}\right) \subseteq\left[i_{1}: i_{2}\right]$, one of the three following conditions hold: (i) $s_{1}\left(\mathrm{~g}_{3}\right) \neq s_{1}\left(\mathrm{~g}_{3}+1\right)$, (ii) $s_{1}\left(\mathrm{~g}_{3}\right)="+"$ and $s_{1}\left(\mathrm{~g}_{3}+1\right)="+"$ and $\left(\mathrm{g}_{3}, \mathrm{~g}_{3}+1\right)$ is not adjoining in $P_{1}$, or (iii) $s_{1}\left(\mathrm{~g}_{3}\right)=$ " - " and $s_{1}\left(\mathrm{~g}_{3}+1\right)=$ " and $\left(\mathrm{g}_{3}+1, \mathrm{~g}_{3}\right)$ is not adjoining in $P_{1}$. Hence, if each such gene $\mathrm{g}_{3}$ satisfies at least one condition, we can define arbitrarily a total order between $i_{1}$ and $i_{2}$ without adding an adjacency.

\section{Experimental results}

Following the example of [4], we have tested our algorithms on simulated data to assess the performance of our programs for different parameters inherent to 
partial orders. The rationale for this choice is two-fold. For one, the presented algorithms are quite complicated and using parameterized data allows us to tune the programs to best solve the problems. For another, using the dataset of [4] makes comparisons easier with previous works. Our linear program solver engine is powered by the minisat+ solver [5]. All computations were carried out on a Duo Intel $\mathrm{CPU} \mathrm{GHz}$ with $2 \mathrm{~Gb}$ of memory running under Linux. The reference data set is from [4]. Blin et al. have generated partial orders $P_{x}$ according to three parameters: the size $n$, the order rate $p$ that determines the number of adjoining in the expression, and the gene distribution rule $q$ that corresponds to the probability of possible adjacencies with respect to the identity. We use 19 different instances for each triplet of parameters $(n, p, q)$ with $n \in\{30,40,50,60,70,80,90\}, p \in\{0.7,0.9\}$ and $q \in\{0.4,0.6,0.8\}$, so that we have 798 genomes for each program. The results of the three programs on this dataset are presented below. We evaluated two criteria: the running time and the given measure (number of common intervals or number of adjacencies) induced by the returned linear extension. The width of partial orders will be also considered. Then, we study the advantages of program Adj-1PO to Adj-2PO for the comparison of a partial order and the identity.

Results for CI-1PO. For program CI-1P0, the minisat+ engine resolves 264 instances out of 342 inputs (77.2\%) with $n \in\{30,40,50\}, p \in\{0.7,0.9\}$, and $q \in\{0.4,0.6,0.8\}$ (Table 1) in less than 5 hours. The 83 remaining cases have been stopped after 30 minutes. We note that CI-1PO reaches its limits even for small instances. As shown in Table 2, the width of an instance is an important parameter that certainly contributes to the complexity of the instances. Indeed, we remark that the number of obtained results decreases according to the width of the instances. In the same way, the average running time increases with the width. We also notice that the width is correlated with the complexity of the problem, which is not surprising since combinatorial difficulty is clearly contained in the greatest sets of non-comparable genes.

\begin{tabular}{|c|c|c|c|}
\hline Size & Number of results & Number of instances & Running time \\
\hline 30 & 95 & 114 & $1 \mathrm{~h} 48 \mathrm{~m}$ \\
\hline 40 & 85 & 114 & $1 \mathrm{~h} 19 \mathrm{~m}$ \\
\hline 50 & 79 & 114 & $1 \mathrm{~h} 44 \mathrm{~m}$ \\
\hline \hline Total & 259 & 342 & $4 \mathrm{~h} 41 \mathrm{~m}$ \\
\hline
\end{tabular}

Table 1. Results of CI-1PO obtained in less than thirty minutes.

Results for Adj-1PO. We applied Adj-1PO on 19 genomes for each triplet $(n, p, q)$ with $n \in\{30,40,50,60,70,80,90\}, p \in\{0.7,0.9\}$ and $q \in\{0.4,0.6,0.8\}$. We obtained 778 results out of $798(97,5 \%)$ after 2 months and 13 days. Due to huge memory requirements, a Quadri Intel(R) Xeon(TM) CPU 3,00 GHz with 


\begin{tabular}{|c||c|c|c|c|c|c|c|c|c|c|c|}
\hline Width & 1 & 2 & 3 & 4 & 5 & 6 & 7 & 8 & 9 & 10 & 11 \\
\hline \hline Number of instances & 5 & 63 & 63 & 50 & 44 & 31 & 26 & 18 & 13 & 7 & 9 \\
\hline Number of solved instances & 5 & 63 & 63 & 44 & 36 & 26 & 15 & 5 & 1 & 1 & 0 \\
\hline \% of solved instances & $100 \%$ & $100 \%$ & $100 \%$ & $88 \%$ & $82 \%$ & $84 \%$ & $58 \%$ & $28 \%$ & $8 \%$ & $15 \%$ & $0 \%$ \\
\hline Average running time (sec) & 2 & 13 & 28 & 60 & 88 & 154 & 201 & 311 & 73 & 490 & \\
\hline
\end{tabular}

Table 2. Impact of width for CI-1PO.

$16 \mathrm{~Gb}$ of memory running Linux was required for 14 of the runs. We note that parameter $p$ has the largest impact on the running time. It is indeed significantly higher when $p=0.7$ rather than when $p=0.9$, i.e., when the partial orders have less adjoining. The width also affects the running time. Parameter $q$ seems, however, to have no impact on the running time. For the 70 cases where the running time exceeds 1 hour, we note that the corresponding genomes contain 50 genes or more, with an order rate $p$ equals to 0.7 (58 times out of 70 ), but without specific gene distribution (21 times $0.4,27$ times 0.6 and 22 times 0.8 ). Their width ranges from 5 to 22 and is on average equal to 11.6. For 19 genomes, the linear program could not been solved by minisat+ because the number of constraints and variables is too large for this solver. For these genomes, we observe that their size is greater than or equal to 70 , that $p$ is equal to 0.7 and that $q$ is variable ( 9 times $0.4,3$ times 0.6 and 8 times 0.8 ). Their width is also large: 17.5 on average (from 11 to 29 ), compared to 6 for all genomes.

Results for Adj-2PO. For each triplet, we compare 19 genomes by pairs. At the present time, we have obtained the results for the following triplets: $\{30,0.7,0.8\}$, $\{30,0.9,0.4\},\{30,0.9,0.6\},\{30,0.9,0.8\},\{40,0.9,0.4\},\{40,0.9,0.6\},\{40,0.9,0.8\}$, $\{50,0.9,0.6\},\{50,0.9,0.8\},\{60,0.9,0.6\},\{60,0.9,0.8\}$, and $\{70,0.9,0.8\}$. Before discussing the 11 triplets for which $p=0.9$, let us look at the results obtained with the triplet $\{30,0.7,0.8\}$. The running time is clearly more important when $p=0.7$ rather than $p=0.9$ (2 days and 21 hours for the triplet $\{30,0.7,0.8\}$ and 40 minutes for the triplet $\{30,0.9,0.8\})$. Moreover, fewer results were obtained (90 results for the triplet $\{30,0.7,0.8\}$ and 171 for the triplet $\{30,0.9,0.8\}$ ). For the 11 triplets for which $p=0.9$, we made 1881 comparisons (19 genomes compared with 18 genomes of the same triplet). We manage to obtain in 1668 results (i.e., $88.6 \%$ ) in a little over 38 days. For the 213 unfinished runs, two types of problems have emerged: either the linear program has too many variables and constraints for the solver minisat+, or the memory requested is too large for the Duo Intel $2 \mathrm{~Gb}$ of memory. The width clearly influences the running time. Indeed, when the sum of width of both partials orders $P_{1}$ and $P_{2}$ is less than 5 , the average running time is 14 seconds, while it is of 56 minutes when this sum is more than 5. Parameter $q$ has an impact on the number of adjacencies only.

Comparison between $A d j-1 P 0$ and $A d j-2 P 0$. To evaluate Adj-1P0, we compare its running time with Adj-2PO when $P_{2}$ is the identity. For the 19 instances of each triplet $(n, p, q)$ such that $n$ is in $\{30,40\}, p$ is in $\{0.7,0.9\}$ and $q$ is in 
$\{0.4,0.6,0.8\}$, the sum of running times is 2 hours and 30 minutes (minimum $=$ 0.1 second, maximum $=15$ minutes $25 \mathrm{~s}$ ) for $\mathrm{Adj}-1 \mathrm{PO}$ and 67 hours $($ minimum $=$ 0.1 second, maximum $=14$ hours $42 \mathrm{~m}$ ) for Adj-2PO. Clearly, Adj-1PO is much faster than Adj-2PO from the viewpoint of running time (a diminution of $96.3 \%$ ). If we compare the running time for each case, we note that both programs have a running time more important for the same genome. We can infer that both programs are facing the same difficulties. This is not surprising in view of the similarities of variables and constraints of linear programming of Adj-1PO and Adj-2PO.

\section{Conclusion and future works}

Our results are quite preliminary and there is still a great amount of work to be done. Among other things, one can cite: (i) for each case, determine other strong and relevant rules for speeding-up the process by avoiding the generation of too many variables and constraints, (ii) generalize the program CI-1PO to compute the number of common intervals between two partial orders, and (iii) define and evaluate heuristics for these problems. Indeed, we do think that our approach is useful for providing exact reference results to which new developed heuristics can be compared.

\section{References}

1. S. Angibaud, G. Fertin, I. Rusu, A. Thévenin, and S. Vialette. Efficient tools for computing the number of breakpoints and the number of adjacencies between two genomes with duplicate genes. J. Computational Biology, 15(8):1093-1115, 2008.

2. S. Angibaud, G. Fertin, I. Rusu, and S. Vialette. A pseudo-boolean general framework for computing rearrangement distances between genomes with duplicates. $J$. Computational Biology, 14(4):379-393, 2007.

3. A. Bergeron and J. Stoye. On the similarity of sets of permutations and its applications to genome comparison. In Proc. 9th International Computing and Combinatorics Conference (COCOON '03), volume 2697 of LNCS, pages 68-79, 2003.

4. G. Blin, E. Blais, D. Hermelin, P. Guillon, M. Blanchette, and N. El-Mabrouk. Gene maps linearization using genomic rearrangement distances. J. Computational Biology, 14(4):394-407, 2007.

5. N. Eén and N. Sörensson. Translating pseudo-boolean constraints into SAT. Journal on Satisfiability, Boolean Modeling and Computation, 2:1-26, 2006.

6. Z. Fu and T. Jiang. Computing the breakpoint distance between partially ordered genomes. J. Bioinformatics and Computational Biology, 5(5):1087-1101, 2007.

7. A. Schrijver. Theory of Linear and Integer Programming. J. Wiley \& Sons, 1998.

8. T. Uno and M. Yagiura. Fast algorithms to enumerate all common intervals of two permutations. Algorithmica, 26(2):290-309, 2000.

9. G. A. Watterson, W. J. Ewens, T. E. Hall, and A. Morgan. The chromosome inversion problem. Journal of Theoretical Biology, 99(1):1-7, 1982.

10. C. Zheng, A. Lenert, and D. Sankoff. Reversal distance for partially ordered genomes. Bioinformatics, 21(1):502-508, 2005. 